\title{
Suspected gonadal mosaicism for isochromosomes $18 p$ and $18 q$ unsubstantiated by fluorescence in situ hybridization analysis of sperm
}

\author{
Marc S. Williams, $M D^{1}$, Kevin D. Josephson, $M S^{1}$, Nurcan Gursoy, $M D^{2}$, and Colleen Jackson-Cook, $P h D^{2}$
}

\begin{abstract}
Purpose: A father had two children, one with isochromosome 18p, and another with isochromosome 18q. The father was counseled that he might have gonadal mosaicism for isochromosomes $18 p$ and $18 q$, which could confer a high recurrence risk. Methods: A sperm sample from the father was analyzed with fluorescence in situ hybridization probes for 18p and 18q. Results: More than 1,000 sperm were scored and none were found with two $18 p$ or $18 q$ signals. There were no differences in the father's specimen compared to a control. Conclusions: There was no evidence for gonadal mosaicism. It is important to confirm clinical hypotheses whenever possible. Genetics in Medicine, 2001:3(4):318-320.
\end{abstract}

Key Words: isochromosome, chromosome 18, fluorescence in situ hybridization, sperm, gonadal mosaicism

The occurrence of isochromosomes involving different arms of the same chromosome in a family has not been previously reported in chromosomally normal individuals. While this finding could be coincidental, the father was counseled that he may have mosaicism for $\mathrm{I}(18)(\mathrm{p} 10)$ and $\mathrm{I}(18)(\mathrm{q} 10)$, possibly limited to gonadal tissue. The couple wished to have additional pregnancies, so studies to identify potential mosaicism for an isochromosome 18 were done on a sperm sample from the father using fluorescence in situ hybridization (FISH) techniques.

\section{METHODS AND RESULTS}

A fetus with multiple congenital anomalies was identified by prenatal ultrasound. Chromosome studies that were done following an amniocentesis identified an abnormal chromosomal complement having an isochromosome for the long arm of chromosome 18, [46,XY,I(18)(q10)], which was found in all cells. The baby was born at term and died shortly after birth. An autopsy confirmed multiple anomalies, which included holoprosencephaly, with cyclopia and proboscis; large omphalocele; ventricular septal defect; single-lobed right lung; micromulticystic kidneys; hypoplastic adrenal glands, and multiple accessory spleens. The lymphocyte chromosome analysis of both parents was normal.

A second pregnancy involving the same father and a different mother was evaluated by amniocentesis due to advanced

\footnotetext{
${ }^{1}$ From the Department of Pediatrics, Gundersen Lutheran Medical Center, La Crosse, Wisconsin; ${ }^{2}$ Department of Human Genetics, Medical College of Virginia Campus of Virginia Commonwealth University, Richmond, Virginia.

Marc S. Williams, MD, Department of Pediatrics, Gundersen Lutheran Medical Center, 1836 South Avenue, La Crosse, WI 54601.

Received: March 26, 2001.

Accepted: May 4, 2001.
}

maternal age. In this case, the fetal chromosomal complement showed an additional isochromosome for the short arm of chromosome $18[47, \mathrm{XY},+\mathrm{I}(18)(\mathrm{p} 10)]$. The chromosome of origin was confirmed by $18 \mathrm{p}$ FISH probes, although the probe differed from that used in the spermatic analysis. This finding was confirmed in chorionic villus cells. No fetal anomalies were identified on ultrasound. The pregnancy was electively terminated at 21 weeks. There were no external anomalies. An autopsy was declined. The second mother's chromosomes were normal. A chromosome analysis was repeated for the father, with additional cells being counted to better assess potential mosaicism. The father was again found to be normal $(46, \mathrm{XY})$. No laboratory was found that was willing to perform parent of origin studies on the iso18p chromosome.

The semen sample was collected by masturbation into a sterile plastic container. Upon receipt in the laboratory, the sample was liquefied at $37^{\circ} \mathrm{C}$ and processed according to our modifications of the protocol of Guttenbach and Schmid. ${ }^{1}$ Briefly, the sample was washed in Hank's balanced salt solution, incubated with Carnoy's fixative (3:1 methanol: glacial acetic acid) for 10 minutes at $-20^{\circ} \mathrm{C}$, washed twice with cold fixative, and stored at $-20^{\circ} \mathrm{C}$. Slides were prepared by dropping the diluted sperm suspension onto cleaned, wet microscope slides, which were then heated at $60^{\circ} \mathrm{C}$ for 30 seconds.

Prior to FISH, the sperm slides were pretreated according to our modifications of the protocol of Robbins et al. ${ }^{2}$ First, the sperm slides were digested 20-30 seconds in a 0.03\% trypsin solution in Sorensen's buffer ( $\mathrm{pH}$ 8.5), followed by brief washes in dilute bovine serum albumin and Millipore water. The slides were then incubated for 30 minutes in a $0.01 \mathrm{M}$ dithiothreitol (DTT)/0.1 M Tris ( $\mathrm{pH} 8.0$ ) solution, followed by 90 minutes in a 0.001MDTT/0.01 diiodosalicylate, lithium salt (LIS)/0.1 M Tris ( $\mathrm{pH} 8.0$ ) solution. This pretreatment was followed by a brief rinse in $2 \times$ saline sodium citrate (SSC) $(\mathrm{pH}$ 7.0), 8 minutes in $4 \%$ paraformaldehyde, and two washes (2 
minutes each) in $2 \times$ SSC (all solutions at room temperature). The slides were then allowed to air-dry, dipped into a $0.1 \%$ gelatin solution to enhance signals brightness and reduce nonspecific binding, ${ }^{3}$ and again air-dried.

FISH was performed using subtelomeric probes specific for $18 \mathrm{p}$ (Spectrum Green) and 18q (Spectrum Orange). Immediately after pretreatment, the slides and probes were denatured according to the probe manufacturer's protocol (Vysis, Inc.) with the adaptation of a 10 -minute denaturation time for the sperm chromatin. Following hybridization, the sperm chromatin was counterstained with DAPI, and the fluorescent signals were visualized using a Zeiss epifluorescent Axioskop equipped with single (Spectrum Orange, Spectrum Green; Vysis) and triple band pass filters (Omega). Semen samples from two control individuals, who were of proven fertility, were also collected and processed using this identical protocol.

A total of 1,000 sperm were scored for each male (this value was determined using power calculations and is in keeping with the number of sperm scored in a sperm aneuploidy protocol that is ongoing in the laboratory). Sperm nuclei that were overlapping, disrupted, diffuse, or devoid of signals were not scored. Same color signals connected by any apparent fluorescence were scored as one signal. Disomy for a probe was classified as two signals of the same color, located within the nucleus, that were comparable in size and intensity. When scoring sperm, all focal planes were viewed to ensure that all signals in the three-dimensional sperm head were visualized (Fig. 1).

A total of 3 of the $1,000(0.3 \%)$ sperm scored from the father's sample had an extra chromosome 18 (two 18p signals and two $18 \mathrm{q}$ signals). This value is not significantly different from that of the healthy control subjects $(0.3 \%$ and $0.6 \%)$, who

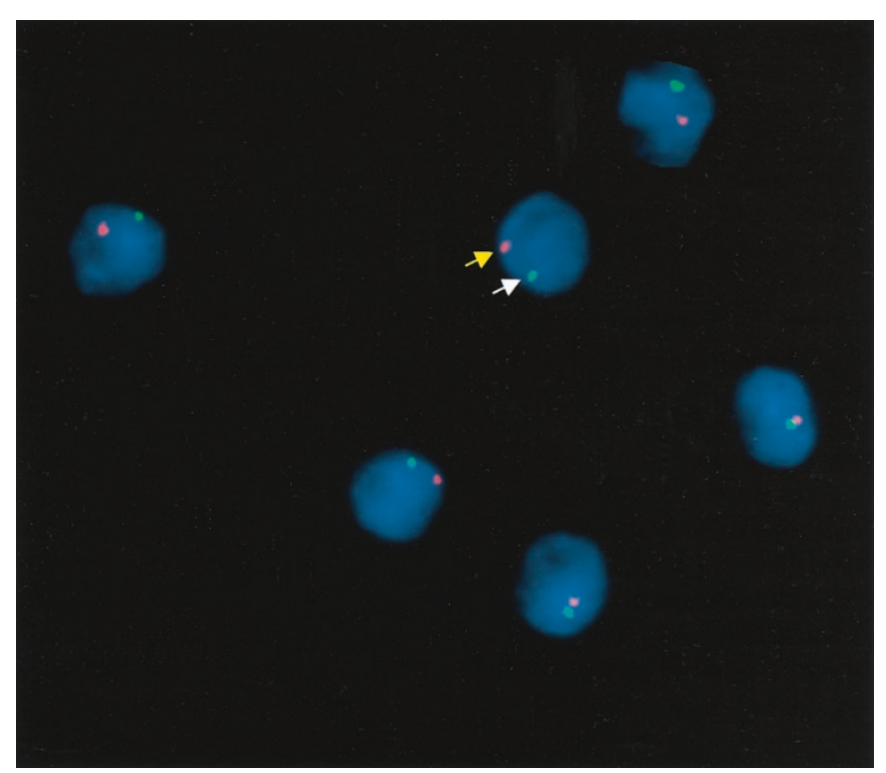

Fig. 1 Representative photomicrograph of sperm following FISH. Each of the six sperm shown has one signal for chromosome 18p (green; highlighted by a white arrow in a centrally located sperm) and one signal for chromosome 18q (orange; highlighted by a yellow arrow in a centrally located sperm). were studied using the same probes. No cells having multiple signals were rejected from either the father or the control males (using the above-specified criteria). None of the sperm in the father's sample had a FISH pattern suggestive of an isochromosome involving chromosome 18 (i.e., 2 signals for $18 \mathrm{p}$ and none for $18 \mathrm{q}$ or 2 signals for $18 \mathrm{q}$ and none for $18 \mathrm{p}$ ). Testing for mosaicism in other cell lines was not performed.

\section{DISCUSSION}

Mosaicism for chromosomal anomalies is a relatively common phenomena. While this is most frequently recognized as a result of lymphocyte chromosome analysis, it has been recognized that mosaicism can disappear from a cell line with rapid turnover, such as blood. ${ }^{4}$ If clinical suspicion is raised about mosaicism, examination of tissue other than blood should be undertaken. Skin fibroblasts are one of the tissues of choice. However, molecular techniques that can be used in nondividing tissues have allowed for analysis of buccal and follicular cells. Clinically, it seemed unlikely that this patient had highlevel mosaicism for either isochromosome, given that he had no physical or cognitive abnormalities. Even if somatic mosaicism had been demonstrated in another tissue, it would not provide an answer regarding gonadal involvement nor would it address the level of gonadal mosaicism present. Recurrence risks would be unable to be quantified. Conversely, absence of somatic mosaicism does not rule out gonadal mosaicism.

Gonadal mosaicism has been postulated as a cause for familial recurrence of autosomal dominant and X-linked disorders, as well as chromosome disorders. Molecular diagnostic techniques have been applied in a minority of cases to confirm both isolated gonadal mosaicism ${ }^{5}$ and generalized somatic mosaicism that includes the gonads. ${ }^{6}$ Molecular analysis of sperm has been used both to rule out ${ }^{5}$ and confirm $^{7}$ gonadal mosaicism for single gene disorders. The latter study did not look at tissues other than lymphocytes to rule out somatic mosaicism, however, this is irrelevant when the concern involves recurrence risk.

Gonadal mosaicism for chromosome anomalies has been confirmed, most frequently in patients with mosaic Turner syndrome, where presence of Y chromosomal material in the gonad has been demonstrated using FISH and other molecular techniques. This mosaicism is not usually limited to the gonad, although there are reports where mosaicism limited to the gonads has been proven. ${ }^{8,9}$ Gonadal mosaicism for trisomy 21 has also been reported in couples having 2 or more trisomic children. ${ }^{10-12}$ However, as in the cases of Turner Syndrome, these rare families typically also have somatic cell mosaicism (albeit in low levels for some cases). In addition, there is a least one report of recurrence of a specific chromosomal rearrangement in three pregnancies, despite normal lymphocyte and fibroblast chromosome analysis_-strongly suggesting mosaicism limited to the gonad. ${ }^{13}$ A single case report of recurrence of isochromosome $18 \mathrm{q}$ in siblings has also been reported in which the parental lymphocyte chromosomes were normal, but no other tissues were evaluated. ${ }^{14}$ Most recently, Boyle et al. ${ }^{15}$ de- 
scribed a very interesting family. In this kindred, the mother of two half-sisters, both of whom had an isochromosome for $18 \mathrm{p}$, was identified (using DNA markers) to have germline mosaicism for an $\mathrm{I}(18)$ (p10) with no evidence of mosaicism in the other tissues tested (blood and fibroblasts). In this male, no sperm were detected that had a FISH pattern suggestive of bearing an isochromosome. Also, the aneuploidy levels for this male were not significantly different from those noted in the control males. Given that the subtelomeric probes used for this study provided information for only one chromosome, the data collected did not allow for a definitive distinction between disomic and diploid sperm. However, the estimates of disomy that were obtained in sperm from these men, using subtelomeric probes $(0.3 \%, 0.3 \%$, and $0.6 \%)$, were not significantly different from the disomy values for chromosome 18 (mean, $0.5 \% \pm 0.05)$ that we noted in a larger study $(N=54 \mathrm{men})$ in our laboratory. In the larger study, we used a three color/chromosome FISH probe combination (including a pericentromeric probe for chromosome 18). ${ }^{16}$ Thus, given the similarity between the disomy values obtained in this and our previous study (in the latter of which we could distinguish disomy from diploidy since three chromosomes were simultaneously scored) it seems likely that the contribution of diploid cells, if any, to the " 2 signals/subtelomeric probe" values in the father is minimal.

It has been recognized in mammals that the germline is temporarily sequestered into extraembryonic regions (specifically the secondary yolk sac in humans), although the reasons for this sequestration are obscure. ${ }^{17}$ This does suggest the possibility that chromosomal mosaicism limited to the gonad could occur. A recent letter by Stavropoulos et al. ${ }^{18}$ provides confirmation of this hypothesis. They report a fetus with confined placental mosaicism for Trisomy 16 (76\% of trophoblast cells and $59 \%$ of stromal cells demonstrated 3 signals for chromosome 16). The pregnancy was electively terminated. Several fetal tissues were examined (oocyte, brain, lung, kidney, and blood) using FISH with probes specific for the centromere of chromosome 16 (with control probe for chromosome 18). Trisomy 16 was not seen in any tissues in excess of controls, with the exception of oocyte, where three chromosome 16 signals were identified in $26 \%$ of these cells. This finding is supported by evidence that suggests that the chorionic stroma of the placenta and the primordial germ cells share common progenitor cells. ${ }^{17}$ The phenomenon of germ line mosaicism was extensively reviewed by Zlotogora. ${ }^{19}$

As with any hypothesis, no matter how compelling, it is important to test the hypothesis, if at all possible. This statement is well illustrated in this case. The hypothesis that our patient had gonadal mosaicism for reciprocal isochromosomes involving chromosome 18 is both plausible and compelling (given only one published case of familial recurrence for isochromosome 18q, implying extreme rarity). New molecular techniques allowed for a test of this hypothesis, with the result that it was not supported. This information led to appropriate counseling regarding recurrence risk, which is believed to be extremely low. Analysis of sperm has a high degree of utility due to the ready availability of these cells without the use of invasive techniques, as well as the large number that are available for analysis. Expansion of this technique, using other molecular methods could allow for quantitative assessment of gonadal mosaicism in males in single gene disorders as well as chromosomal disorders, as in this case. This should allow for a quantitation of the recurrence risk, based on the presence or absence of mosaicism, as well as the level of mosaicism detected.

\section{Acknowledgments}

This work was supported, in part, by NIH grant R01 HD33527.

\section{References}

1. Guttenbach M, Schmid M. Non-isotopic detection of chromosome 1 in human meiosis and demonstration of disomic sperm nuclei. Hum Genet 1991;87:261-265.

2. Robbins WA, Segraves R, Pinkel D, Wyrobek AJ. Detection of aneuploid sperm by fluorescence in situ hybridization: evidence for a donor difference in frequency of sperm disomic for chromosome 1 and Y. Am J Hum Genet 1993;52:799-807.

3. Lakhotia SC, Sharma A, Mutsuddi M, Tapadia MG. Gelatin as a blocking agent in southern blot and chromosomal in situ hybridizations. Trends Genet 1993;9:261.

4. Horn D, Majewski F, Hildebrandt B, Korner H. Pallister-Killian syndrome: normal karyotype in prenatal chorionic villi, in postnatal lymphocytes, and in slowly growing epidermal cells, but mosaic tetrasomy 12p in skin fibroblasts. J Med Genet 1995; 32:68-71.

5. Forissier JF, Richard P, Briault S, Ledeuil C, Dubourg O, Charbonnier B, Carrier L, Moraine C, Bonne G, Komajda M, Schwartz K, Hainque B. First description of germline mosaicism in familial hypertrophic cardiomyopathy. J Med Genet 2000;37: 132-134.

6. Putnam EA, Park ES, Aalfs CM, Hennekam RCM, Milewicz DM. Parental somatic and germ-line mosaicism for a FBN2 mutation and analysis of FBN2 transcript levels in dermal fibroblasts. Am J Hum Genet 1997;60:818-827.

7. Lázaro C, Ravella A, Gaona A, Volpini V, Estivill X. Neurofibromatosis type 1 due to germ-line mosaicism in a clinically normal father. N Engl J Med 1994;331:14031407.

8. Goldstein DE, Kelly TE, Johanson AJ, Blizzard RM. Gonadal dysgenesis with 45,XO/ 46,XX mosaicism demonstrated only in a streak gonad. J Pediatr 1977;60:604-605.

9. Fujimoto A, Ebbin AJ, Towner JW. Isolated gonadal sex chromosome mosaicism in primary amenorrhea. Am J Obstet Gynecol 1978;131:699-700.

10. Jagiello GM, Taylor AI. Personal communication to Hamerton JL, in Human cytogenetics, Vol II, Clinical cytogenetics. New York: Academic Press, p 245, 1971.

11. Hsu LYF, Gertner M, Leiter E, Hirschhorn K. Paternal trisomy 21 mosaicism and Down syndrome. Am J Hum Genet 1971;23:592-601.

12. Parke JC, Grass FS, Pixley R, Deal J. Trisomy 21 mosaicism in two successive generations in a family. J Med Genet 1980;17:48-49.

13. Sachs ES, Jahoda MGJ, Los FJ, Pijpers L, Wladimiroff JW. Trisomy 21 mosaicism in gonads with unexpectedly high recurrence risks. Am J Med Genet Suppl 1990;7:186188.

14. Krüger G, Götz J, Dunker H, Pelz L. Isochromosome (18q) in siblings. Clin Genet 1987;32:249-253.

15. Boyle J, Sangha K, Dill F, Robinson W, Yong SL. Grandmaternal origin of an isochromosome 18p present in two maternal half-sisters. Am J Med Genet 2001;101: 65-69.

16. Winters J, Meyer J, Jackson-Cook C. Assessment of the genetic contribution to sperm aneuploidy levels in humans. Am J Hum Genet 1996;59:A136.

17. Buehr M. The primordial germ cells of mammals: some current perspectives. Exp Cell Res 1997;232:194-207.

18. Stavropoulos DJ, Bick D, Kalousek DK. Molecular cytogenetic detection of confined gonadal mosaicism in a conceptus with trisomy 16 placental mosaicism. Am J Hum Genet 1998;63:1912-1914.

19. Zlotogora J. Germ line mosaicism. Hum Genet 1998;102:381-386. 